Société d'histoire de la révolution de 1848 et des

révolutions du XIXe siècle

$30 \mid 2005$

Pour une histoire culturelle de la guerre au XIXe siècle

John M. Knapp, Behind the Diplomatic Curtain: Adolphe de Bourqueney and French Foreign Policy, 1816-1869, Ohio, University of Akron Press, 2001, xvi + 343 p. ISBN : $1884836712.49,95$ dollars.

David Brown

\title{
OpenEdition
}

Journals

Édition électronique

URL : http://journals.openedition.org/rh19/1045

DOI : $10.4000 /$ rh 19.1045

ISSN : $1777-5329$

Éditeur

La Société de 1848

Édition imprimée

Date de publication : 1 juin 2005

ISSN : 1265-1354

Référence électronique

David Brown, «John M. Knapp, Behind the Diplomatic Curtain : Adolphe de Bourqueney and French Foreign Policy, 1816-1869, Ohio, University of Akron Press, 2001, xvi + 343 p. ISBN : 1884836712 49,95 dollars. », Revue d'histoire du XIXe siècle [En ligne], 30 | 2005, mis en ligne le 19 février 2006, consulté le 22 septembre 2020. URL : http://journals.openedition.org/rh19/1045 ; DOI : https:// doi.org/10.4000/rh19.1045

Ce document a été généré automatiquement le 22 septembre 2020.

Tous droits réservés 


\section{John M. Knapp, Behind the Diplomatic Curtain: Adolphe de Bourqueney and French Foreign Policy, 1816-1869, Ohio, University of Akron Press, 2001, xvi +343 p. ISBN : 1884836712 . 49,95 dollars.}

\section{David Brown}

1 La carrière diplomatique d'Adolphe de Bourqueney (1799-1869) a vraiment été l'une des plus intéressantes du XIX ${ }^{\mathrm{e}}$ siècle. Il a eu une vie variée : des États-Unis à Vienne, en passant par Londres et Constantinople, sa carrière était non seulement loin d'être monotone mais elle l'a en plus placé au cœur du monde diplomatique. Bourqueney, né dans le monde aristocratique et donc introduit très tôt dans le monde de la haute politique, a d'abord été nommé troisième secrétaire du ministre français aux États-Unis en 1816, alors qu'il n'avait que seize ans. C'était là le début d'une carrière glorieuse qui l'a conduit, successivement, à Londres (1819-23), Bern (1823-24), Paris (1830-35), Londres de nouveau (1835-41), Constantinople (1841-48) et, finalement, Vienne (1853-59). Son expérience grandit de même que s'accroît son influence sur la politique étrangère de la France, voire sur celle d'autres pays.

2 John Knapp, lui même apparenté (par alliance) à la famille Bourqueney, a produit une étude de grande valeur, basée sur des archives de Bourqueney largement inexploitées jusque là. La collection de papiers est évidemment vaste (Bourqueney aurait décidé dans sa jeunesse de créer une " encyclopédie volumineuse » de sa vie) et John Knapp l'a utilisée pour élaborer un compte rendu de cette vie. Quand il avait seize ans, Bourqueney est allé à Washington pour commencer sa carrière et c'est cette période que John Knapp a choisie comme point de départ de son étude. À partir de là le livre fournit une biographie conventionnelle : ce que Bourqueney a rencontré et ce qu'il en a 
pensé. Le récit de ses affaires diplomatiques des années 1810 aux années 1860 est déjà connu et John Knapp n'apporte que peu d'éléments nouveaux. Mais il ne s'agit là que d'une partie de l'étude. Comme le laisse entendre le titre, John Knapp offre un aperçu des ficelles du monde diplomatique. Il propose ainsi des observations sur les conditions et le mode de vie dans les villes et les pays dans lesquels Bourqueney a travaillé, il s'attache par exemple à la description des mauvaises conditions de la vie viennoise. Il tente également de considérer l'influence personnelle de son personnage. À son avis, il est important de souligner que les diplomates travaillaient «dans un contexte humain ». À Washington et à Londres, au commencement de sa carrière, Bourqueney s'est ainsi rapidement fait la réputation d'un homme "en pleine ascension » et il a notamment développé un bon rapport avec le vicomte Palmerston, aidant le ministre britannique, par exemple, dans sa politique sur l'Empire ottoman des années 1830. Lors des profonds changements du gouvernement français entre le début et la fin de sa carrière, Bourqueney a maintenu une position centrale mais la période la plus heureuse pour Bourqueney a sans doute été celle de la Monarchie de Juillet. C'est en effet à cette époque qu'il est devenu "porte-parole de la cour dans le domaine de la politique étrangère et la diplomatie " puis il a poursuivi son ascension en se faisant nommer ambassadeur à Constantinople. Par ailleurs, Bourqueney est un homme curieux, que certains désignent même comme une girouette, ce qui le rend capable de s'adapter et d'être aussi à l'aise dans la France de Louis xviii que dans celle des Orléans ou celle de Napoléon III. Il aurait été ainsi intéressant que John Knapp analyse les idées et la conception politique de Bourqueney, afin d'expliquer son adaptation aux différents milieux politiques et aux divers gouvernements français parce qu'il est évident que son point de vue a influencé sa conduite de la diplomatie. S'il est certain que les affaires étrangères sont le produit des actions humaines, comme John Knapp le soutient, on aurait alors aimer connaître les opinions politiques de Bourqueney au delà de l'histoire de ses expériences vécues et de ses rencontres.

Il est clair que Bourqueney a eu un impact profond grâce à sa capacité de travailler en harmonie avec ses contemporains. Retracer l'histoire personnelle d'un tel diplomate est donc utile pour comprendre la nature des relations internationales, mais l'auteur conserve peut-être un peu trop d'empathie à l'égard de son sujet. Il s'appuie de manière un peu excessive sur le point de vue de son personnage et, de temps en temps, cette biographie tend vers l'hagiographie. Dès l'introduction, John Knapp considère d'ailleurs son projet comme un exercice, ayant en partie pour but d'affirmer l'importance de Bourqueney. Et au final, Knapp conclut que «tout bien considéré, on apprécie Adolphe » Bourqueney, un homme honnête et impartial. Ce ton toujours favorable à Bourqueney est une constante du livre. Le dernier chapitre nous affirme même que le diplomate "nous parle aujourd'hui ", que sa vie et sa carrière nous offre une série de leçons. Par exemple, John Knapp explique que, selon Bourqueney, les diplomates doivent être flexibles, bien formés, fidèles à la réalité de leurs rapports et qu'ils doivent favoriser les rencontres en face à face, etc. Il s'agit là de réflexions un peu minces et manquant d'originalité, ce qui diminue l'impact de l'étude. Ceci mis à part, l'étude de John Knapp est une étude valable qui représente une lecture utile pour qui souhaite découvrir le monde de la diplomatie vu par un personnage qui a travaillé en arrièreplan mais qui a eu un impact profond sur les relations entre la France et le reste du monde. Par ailleurs, on a ici l'opportunité de lire l'histoire politique de la France pendant une époque de bouleversements. 
4 Behind the Diplomatic Curtain représente un guide efficace des archives de Bourqueney et dresse le portrait d'un homme fréquemment, et injustement, perdu de vue. Si le ton n'est pas toujours suffisamment critique, il est pourtant vrai que cette étude met l'accent sur le rôle d'un homme important qui a traversé les mondes et les périodes du $\mathrm{XIX}^{\mathrm{e}}$ siècle sans difficulté, et elle souligne en même temps la richesse des archives de Bourqueney.

\section{AUTEUR}

DAVID BROWN

Université de Strathclyde, Glasgow, Grande-Bretagne 